\title{
Standardizing the Protocols for Enhanced Recovery From Colorectal Cancer Surgery: Are We a Step Closer to Ideal Recovery?
}

\author{
Mosab Shetiwy, Tamer Fady, Fayez Shahatto, Ahmed Setit \\ Department of Surgery, Surgical Oncology Unit, Oncology Center, Mansoura University (OCMU), Mansoura, Egypt
}

Purpose: Enhanced recovery protocols are being implemented into the standard of care in surgical practice. This study aimed to insert a steadfast set of elements into the perioperative care pathway to establish an improved recovery program for colorectal cancer patients.

Methods: Seventy patients planned for elective laparoscopic colorectal resection were randomized into 2 groups: conventional recovery group $(n=35)$ and enhanced recovery group $(n=35)$. The primary outcome was the length of hospital stay. Secondary outcomes included the times of removal of nasogastric tubes (NGTs), successful enteral feeding, and removal of drains, postoperative complications, intra-hospital mortality, and rate of readmission.

Results: The mean postoperative hospital stay was $4.49 \pm 0.85$ days vs. $13.31 \pm 6.9$ days $(\mathrm{P}<0.001)$, the mean time of removal of NGTs was $0.77 \pm 1.031$ days vs. $3.26 \pm 2.737$ days $(\mathrm{P}<0.001)$, the mean time of successful enteral feeding was $1.89 \pm 1.13$ days vs. $5.46 \pm 1.67$ days $(\mathrm{P}<0.001)$, and the mean time for removal of intra-abdominal drains was $2.94 \pm$ 1.056 days vs. $9.06 \pm 3.757$ days $(\mathrm{P}<0.001)$ for the enhanced and the conventional groups, respectively. Complications were significantly lower among patients in the enhanced group $(25.7 \%$ vs. $65.7 \%)(\mathrm{P}=0.001)$. The rates of readmission were similar in the 2 groups.

Conclusion: Applying definite evidence-based elements to the colorectal rehabilitation program significantly boosts the recovery pathway with favorable outcomes, including faster recovery of gastrointestinal tract functions, lower morbidities, and eventually earlier discharge from the hospital.

Keywords: Perioperative care; Colorectal surgery; Laparoscopy; Fast track surgery; Prospective studies

\section{INTRODUCTION}

In order to handle the metabolic response of surgery and speed up the recovery of patients, several enhanced recovery protocols have been formulated with encouraging results. Reduced incidence of postoperative morbidity and mortality, shorter hospital

Received: February 11, 2017 - Accepted: March 10, 2017

Correspondence to: Mosab Shetiwy, M.D., MRCS

Department of Surgery, Surgical Oncology Unit, Oncology Center, Mansoura University (OCMU), Mansoura, Egypt

Tel: +20-1000360060, Fax: +20502238673

E-mail: mosabsaad@mans.edu.eg

(c) 2017 The Korean Society of Coloproctology

This is an open-access article distributed under the terms of the Creative Commons Attribution NonCommercial License (http://creativecommons.org/licenses/by-nc/4.0) which permits unrestricted noncommercial use, distribution, and reproduction in any medium, provided the original work is properly cited. stay, as well as reduction in healthcare costs were among these results [1]. The steps to achieve an accelerated recovery begin with a thorough preoperative assessment in the first clinic encounter embedded with appropriate patient education and counseling with a physiotherapist and a stoma nurse when feasible. The prolonged period of preoperative overnight fasting is replaced with carbohydrate loading with drinks up to 4 hours prior to induction of anesthesia. For pain management, epidural analgesia is treasured. Careful fluid therapy is essential. Early 'enforced' ambulation, enteral nutrition and rehabilitation are encouraged postoperatively $[2,3]$.

However, different versions of the enhanced recovery protocols have been established by combining various sets of elements, and no set is considered an 'ideal' protocol of enhanced recovery; thus, authors all around the world have been utilizing a series of programs that focus on specific key components to improve the re- 
covery pathway [4]. This study was designed to implement the concept of fast track care and to set a policy of enhanced recovery after surgery protocols into the surgical management of patients with a colorectal carcinoma at our oncology center. All procedures performed in this study were in accordance with the ethical standards of the institutional and/or national research committee and with the 1964 Helsinki Declaration and its later amendments or comparable ethical standards. Informed consent was obtained from all individual participants included in the study.

\section{METHODS}

\section{Patients}

Seventy colorectal cancer patients planned for elective resection were admitted to the Surgical Oncology Unit, Oncology Center Mansoura University (OCMU) between June 2012 and October 2016. Patients were randomized into 2 groups: 35 patients underwent a conventional recovery pathway and 35 patients underwent an enhanced recovery pathway. Inclusion criteria for participants were the presence of a pathologically confirmed colorectal carcinoma amenable for elective surgery and no severe physical disability (American Society of Anesthesiologists physical status classification I-III). Exclusion criteria included a previous history of abdominal surgery, chronic pain syndrome, and the need for emergency surgery. All patients complied with the procedures after having given written informed consent. This controlled clinical trial was approved by the 'Institutional Review Board' of the Faculty of Medicine, Mansoura University.

\section{Preoperative preparations}

Standard preoperative health education was delivered to patients within the conventional group while 'individualized' counseling about the proposed fast track care plan (Table 1) and about the daily chores, milestones and expectations regarding the procedure was delivered to patients within the enhanced recovery group. Routine bowel cleansing was done for patients in the conventional group while selective mechanical bowel preparation was performed for patients in the enhanced group (for left colonic and rectal lesions). Prophylaxis against thromboembolism was mandatory in all patients in both groups of this study (special deterrent stockings and a single dose of low molecular weight Heparin [LMWH] in the evening before surgery - 6:00 PM).

Patients in the conventional recovery group were instructed to take clear fluids on the day before surgery and to fast overnight

Table 1. Perioperative care pathway

\begin{tabular}{|c|c|c|}
\hline Element & Conventional care (group 1) & ERAS (group 2) \\
\hline Preoperative counseling & Done by surgeons & Done by ERAS team \\
\hline \multirow[t]{2}{*}{ Preoperative fasting } & Drinks on the 2 days before surgery & Carbohydrate-rich drinks on the day before surgery \\
\hline & Fasting from the night of surgery & $\begin{array}{l}\text { Drinking is encouraged until } 4 \text { hours preoperatively } \\
\text { (morning of surgery) }\end{array}$ \\
\hline Preoperative mechanical bowel preparation & Done unless refused & Only for rectal/rectosigmoid malignancy \\
\hline Perioperative fluid management & Not changed & Yes (avoidance of sodium/fluid overload) \\
\hline $\begin{array}{l}\text { Assisted laparoscopic surgery with small transverse/ } \\
\text { longitudinal incisions }\end{array}$ & $\begin{array}{l}\text { Type of incision used according to surgeon's } \\
\text { preference }\end{array}$ & Preference for transverse incisions over longitudinal incisions \\
\hline Intraoperative warming of patients and IV fluids & Not mandatory & Mandatory \\
\hline Nasogastric tube removal & Only when peristalsis occurred & On the day of surgery (POD 0) except for patients with PONV \\
\hline \multirow[t]{2}{*}{ Time of starting oral nutrition } & $\begin{array}{l}\text { Not before } 3 \text { days } \mathrm{PO} \text { once peristalsis occurs } \\
\text { (motion or flatus) }\end{array}$ & Oral sips within 24 hours of surgery \\
\hline & & $\begin{array}{l}\text { Then, resume full diet on POD } 3 \text { with IV fluid restricted to a } \\
\text { minimum }\end{array}$ \\
\hline Postoperative ambulation & Patients gets out of bed on POD 1 & $\begin{array}{l}\text { Forcing patients to get out of bed for } 2 \text { hours postoperatively } \\
\text { (on POD 0) and on the morning of POD } 1\end{array}$ \\
\hline \multirow[t]{2}{*}{ Epidural analgesia } & $\begin{array}{l}\text { Opiates allowed unless contraindicated } \pm \text { IV } \\
\text { NSAIDs }\end{array}$ & Opiates not allowed \\
\hline & & Epidurals for 48 hours only PO \\
\hline \multirow[t]{2}{*}{ Oral analgesia } & Late & Early \\
\hline & After the patient starts oral intake & $\begin{array}{l}\text { Oral regular doses (acetaminophen + NSAIDs) after } 48 \text { hours } \\
\text { PO }\end{array}$ \\
\hline Stimulation of gut motility & Allowed unless refused & Allowed (oral/rectal laxatives) \\
\hline
\end{tabular}

ERAS, enhanced recovery after surgery; IV, intravenous; PO, postoperative; POD, postoperative day; PONV, postoperative nausea and vomiting; NSAID, nonsteroidal antiinflammatory drug. 
until the time of surgery. On the contrary, patients within the enhanced program were supplemented with carbohydrate-rich drinks (3 sachets of Biogainers [Bio Pharma, Giza, Egypt] daily or a similar carbohydrate-rich fluid; Nutrition Advanced Formula [Prime Pharmaceuticals, 10th of Ramadan, Egypt]) starting 2 days before surgery and continuing up to four hours prior to induction of anesthesia to maintain them in a well-fed state.

\section{Operative technique}

A standardized anesthetic protocol was applied to all patients. No preanesthetic medications were allowed in the fast track group. Just before induction of anesthesia, all patients, unless they had refused, had low thoracic epidural catheters (T10-12) inserted for postoperative pain control. Similar laparoscopic techniques were advocated in both groups with preference to smaller transverse incisions in assisted resections within the enhanced recovery group. In the enhanced group, active measures, including warming of the infused intravenous fluids and, in some instances, warming of the air in the operating room, were taken to avoid hypothermia. Warmer coats or blankets were also used for 2 hours postoperatively. Nasogastric tubes (NGTs), urinary catheters and intra-abdominal drains were routinely inserted for patients in both groups.

\section{Postoperative care}

All patients after surgery were transferred according to their vital signs and pre-existing comorbidities either to the intensive care unit, the high dependency unit, or the normal ward for close monitoring of their vital signs and for proper pain management. The patients in the conventional group were administered a combination of a local anesthetic (bupivacaine $0.125 \%$ ) and an opiate (morphine, pethidine, or fentanyl) in epidurals with or without dermal patches (Duragesic [Janssen Pharmaceutica, Beerse, Belgium] patch) unless contraindicated, in which case, the patients were only given analgesic doses of nonsteroidal anti-inflammatory drugs (NSAIDs) at timed intervals. Patients in the enhanced recovery group were not allowed to receive opiates in their epidurals during the postoperative period, but were given intravenous acetaminophen ( $4 \mathrm{~g} /$ day) throughout the recovery period. For breakthrough pain episodes, combinations of NSAIDs and acetaminophen were administered. This combination continued to offer pain relief after removal of the epidurals (48 hours from the surgery).

Laxatives were used on the same day of surgery in the enhanced group while in the conventional group, they were used only when peristalsis occurred. In the conventional group, patients were allowed to get out of bed on the night of surgery or the next morning, if tolerable. In the enhanced recovery group, enforced mobilization started on the day of surgery (postoperative day [POD] 0) for 2 hours and gradually increased to 6 hours by the time of discharge. In the enhanced recovery group, urinary catheters were usually removed on POD 0 unless ureteric/bladder injury had oc- curred (continuous drainage for 14 days). Similarly, NGTs were removed on POD 0 unless severe postoperative nausea and vomiting (PONV) occurred.

In the conventional recovery group, patients were allowed to start oral intake once they had passed motion or flatus (later than POD 3). On the contrary, in the enhanced recovery group, patients were instructed to start oral sips of a nutritional supplement as soon as POD 1 in gradually increasing amounts until permission was given for a normal meal, usually on POD 3, with intravenous fluid being restricted to a minimum or discontinued. After full oral intake had been maintained in both groups, drains were removed, and patients were ready to be discharged. Predetermined discharge criteria were similar for the 2 studied groups: no complications at time of discharge, tolerance to food intake (solid diet) with normalization of gastrointestinal tract functions, successful oral analgesia, independent mobilization, and acceptance (consent) of hospital discharge. Fulfilling these criteria allowed 'safe' hospital departure. A follow-up date 1 week after the patient's discharge from the hospital was given to all patients. $\mathrm{Pa}$ tients were readmitted when they presented on their follow up date or sooner with a surgical complication that could not be managed in the outpatient clinic, i.e., persistent PONV, severe urinary tract infection (UTI), postoperative ileus, and intra-abdominal collection on follow-up ultrasound.

\section{Statistical analysis}

Data were analyzed using IBM SPSS Statistics ver. 23.0 (IBM Co., Armonk, NY, USA). Numerical data were expressed in the form of means \pm standard deviations, and comparisons between groups were made using the chi-square test or Fisher exact test while for categorical data independent samples, the t-test was used. A Pvalue less than 0.05 was considered significant.

\section{RESULTS}

Demographic data regarding patients' age, sex, and location of the tumor (Table 2) were similar in the 2 study groups. Operative techniques were standardized with subsequent similar durations of surgery, types of laparoscopic surgery, and types of incisions utilized in assisted laparoscopies (Table 3).

Patients in the enhanced recovery group were compliant to enforced mobilization within 24 hours after surgery (POD 0) while only five patients in the control group were ambulated successfully within that period $(\mathrm{P}<0.001)$. Similarly, patients in the enhanced recovery group tolerated earlier oral feeding (fluids) without severe PONV $(\mathrm{P}<0.001)$. The times of removal of urinary catheters were similar in the 2 groups $(P=0.202)$. As the removals of NGTs and intra-abdominal drains were linked to successful oral feeding, noticeable significance was noted as regards their times of removal $(\mathrm{P}<0.001)$. Subsequently, enhanced recovery patients met the pre-emptive discharge criteria earlier and thus left the hospital earlier $(\mathrm{P}<0.001)$ (Table 4$)$. 
The readmission rates were similar in the 2 groups (11.4\% each) (Table 4). Four patients in the conventional group were readmitted: one for urine leak (managed by re-exploration and repair), another 2 for anastomotic 'intestinal' leakage (managed by re-exploration and repair), and the last for a complete bursting of the abdominal wound, which required debridement and closure. The enhanced recovery group also had 4 readmissions: 2 for abdominal collections (managed by tubal drainage), another for a UTI and urine retention (managed by catheterization and urine cultures), and the last for severe late-onset PONV (managed conservatively without any further surgical intervention).

Surgical complications are presented in Table 5. Regarding postoperative complications, patients in the enhanced recovery group had lower incidences of complications some of which were statistically significant, such as wound infection $(\mathrm{P}<0.001)$, respiratory tract infections $(\mathrm{P}=0.025)$ and anastomotic leak $(\mathrm{P}=0.05)$. Two

Table 2. Patients' demographics

\begin{tabular}{lccc}
\hline Parameter & $\begin{array}{c}\text { Conventional recovery } \\
(\text { group 1) } \\
(\mathrm{n}=35)\end{array}$ & $\begin{array}{c}\text { ERAS } \\
(\text { group 2) } \\
(\mathrm{n}=35)\end{array}$ & P-value \\
\hline Age (yr) & $53.63 \pm 11.5$ & $48.54 \pm 12.29$ & 0.358 \\
Sex, male : female & $24: 11: 00$ & $21: 14$ & 0.618 \\
Location of the tumor & & & 0.268 \\
Cecum & $3(8.6)$ & $4(11.4)$ & \\
Right & $5(14.3)$ & $8(22.9)$ & \\
Transverse & $1(2.9)$ & $2(5.7)$ & \\
Left & $3(8.6)$ & $7(20)$ & \\
Sigmoid & $5(14.3)$ & $1(2.9)$ & \\
Rectosigmoid & $5(14.3)$ & $2(5.7)$ & \\
Rectum & $12(34.3)$ & $9(25.7)$ & \\
Anorectum & $0(0)$ & $2(5.7)$ & \\
Multiple sites & $1(2.9)$ & $0(0)$ & \\
\hline
\end{tabular}

Values are presented as mean \pm standard deviation or number (\%).

ERAS, enhanced recovery after surgery. patients in the conventional recovery group died of pulmonary artery embolism within the first 30 days following surgery (intrahospital mortality) while the enhanced group showed no intrahospital mortality $(\mathrm{P}=0.493)$.

\section{DISCUSSION}

Metabolic response to injury has been an intriguing topic to surgeons over time. With an aim to lessen the postoperative morbidity and mortality, a pathway was developed for the patients to allow them to benefit from an understanding of the body's response to surgical trauma in order to accelerate that response [5]. Ongoing clinical trials have explored many of the components of these fast track programs with the aim of formulating a standard protocol for enhancing the recovery and thus minimizing the surgical trauma. Several recent randomized trials have reported the use of 4 to 12 enhanced recovery elements, with a mean of 9 , following

Table 3. Surgical procedures

\begin{tabular}{lccc}
\hline Parameter & $\begin{array}{c}\text { Conventional recovery } \\
(\text { group 1) } \\
(\mathrm{n}=35)\end{array}$ & $\begin{array}{c}\text { ERAS } \\
(\text { group 2) } \\
(\mathrm{n}=35)\end{array}$ & P-value \\
\hline $\begin{array}{l}\text { Duration of surgery (min) } \\
\text { Laparoscopic resection }\end{array}$ & $280.71 \pm 69.749$ & $264.43 \pm 53.231$ & 0.276 \\
$\quad$ Totally laparoscopic & $5(14.3)$ & $9(25.7)$ & 0.155 \\
$\begin{array}{l}\text { Laparoscopic resection } \\
\text { with extracorporeal } \\
\text { anastomosis }\end{array}$ & $16(45.7)$ & $19(54.3)$ & \\
$\quad$ Assisted laparoscopic & $14(40)$ & & \\
Incisions used & & $7(20)$ & \\
$\quad$ Longitudinal & $22(62.9)$ & $18(51.4)$ & \\
Transverse & $8(22.9)$ & $8(22.9)$ & \\
No incision & $5(14.3)$ & $9(25.7)$ & \\
\hline
\end{tabular}

Values are presented as mean \pm standard deviation or number (\%). ERAS, enhanced recovery after surgery.

Table 4. Postoperative recovery parameters

\begin{tabular}{|c|c|c|c|}
\hline Parameter & $\begin{array}{l}\text { Conventional recovery } \\
\text { (group 1) }(n=35)\end{array}$ & $\begin{array}{c}\text { ERAS } \\
\text { (group 2) }(n=35)\end{array}$ & P-value \\
\hline No. of patients undergoing early 'enforced' ambulation & $5(14.3)$ & $35(100)$ & $<0.001$ \\
\hline Time of removal of urinary catheters (day) & $2.66 \pm 3.077$ & $1.57 \pm 3.92$ & 0.202 \\
\hline Time of removal of nasogastric tubes (day) & $3.26 \pm 2.737$ & $0.77 \pm 1.031$ & $<0.001$ \\
\hline No. of readmissions & $4(11.4)$ & $4(11.4)$ & 1.000 \\
\hline
\end{tabular}

Values are presented as mean \pm standard deviation or number (\%).

ERAS, enhanced recovery after surgery. 
Table 5. Complications within both groups of the study

\begin{tabular}{lccc}
\hline Parameter & $\begin{array}{c}\text { Conventional } \\
\text { recovery (group 1) } \\
(\mathrm{n}=35)\end{array}$ & $\begin{array}{c}\text { ERAS } \\
\text { (group 2) } \\
(\mathrm{n}=35)\end{array}$ & P-value \\
\hline Nausea and vomiting (PONV) & $5(14.3)$ & $3(8.6)$ & 0.71 \\
Postoperative ileus & $5(14.3)$ & $2(5.7)$ & 0.428 \\
Anastomotic leak & $7(20.0)$ & $1(2.9)$ & 0.05 \\
Wound infection & $11(31.4)$ & $2(5.7)$ & $<0.001$ \\
Respiratory tract infection & $6(17.1)$ & $0(0)$ & 0.025 \\
Intra-abdominal collection & $3(8.6)$ & $2(5.7)$ & 1.000 \\
Urine retention & $1(2.9)$ & $1(2.9)$ & 1.000 \\
Pulmonary embolism & $3(8.6)$ & $0(0)$ & 0.239 \\
Urinary tract infection & $0(0)$ & $2(5.7)$ & 0.493 \\
Acute abdomen & $1(2.9)$ & $0(0)$ & 1.000 \\
Wound bursting & $1(2.9)$ & $0(0)$ & 1.000 \\
UB tear & $0(0)$ & $2(5.7)$ & 0.493 \\
Ureteric leak & $1(2.9)$ & $1(2.9)$ & 1.000 \\
\hline
\end{tabular}

Values are presented as number (\%).

ERAS, enhanced recovery after surgery. PONV, postoperative nausea and vomiting; UB, urinary bladder.

colorectal resections [6]. Kehlet and Wilmore [7] for example proposed a protocol with 15 elements while Wind et al. [1] used 17 elements in their systemic review. Fifteen items were formulated into an enhanced recovery protocol in our study.

Our study showed that employing enhanced recovery protocols helped in boosting the recovery pathway with favorable outcomes, including faster return of GIT functions, reduced postoperative morbidities, and an earlier hospital discharge, after a resection of colorectal cancer. Enhanced recovery trials in the literature show a noticeable reduction in the duration of postoperative hospital stay. The enhanced recovery group in our study had a mean hospital stay of 4.5 days $(\mathrm{P}<0.001)$ compared to the mean hospital stays reported by Wang et al. [8] in China ( $5.1 \pm 3.1$ days, $\mathrm{P}=$ 0.001), Šerclová et al. [9] in the Czech Republic (7.4 \pm 1.3 days, $\mathrm{P}<$ 0.001 ), Vlug et al. [10] in the Netherlands (mean, 5 days; $\mathrm{P}<$ $0.001)$, García-Botello et al. [11] in Spain ( $4.15 \pm 2.2$ days, $\mathrm{P}<$ 0.001 ) and lastly Greco et al. [12] in Italy who analyzed the outcome of 16 clinical trials (mean, 5.8 days) [8-12].

Preoperative counseling has been considered an indispensible factor by some authors [8]. Education and counseling stimulates patients to actively participate in the different tasks offered to them, such as early food intake and early ambulation postoperatively, and helps reduce the psychological stress and fear of the suggested procedures. Highlighted counseling of patients preoperatively are also a requirement of the enhanced program in the protocol guidelines carried out in the United Kingdom, France, Italy and Sweden [13-16].

Late removal of NGTs was considered common practice follow- ing GIT surgery because of the belief that those tubes facilitated gastric emptying, helped avoid pulmonary aspiration, and decreased the risk of leakage from GIT anastomoses. However, their use in the literature is now decreasing after a meta-analysis done by Verma and Nelson [17] that showed an increased complication rate with their routine use. NGTs were removed in $57.1 \%$ of the patients in the enhanced recovery group on the day of surgery while they were removed in $62.9 \%$ of the patients in the conventional recovery group on POD 3. In our study, an association was found between the time of removal of NGTs in the enhanced recovery group and the duration of hospital stay $(\mathrm{P}=0.032)$, indicating that the earlier the NGTs were removed, the faster the patients in this group were discharged.

One of the important discharge criteria to be fulfilled is sufficient enteral nutrition. In our study, the enhanced group patients were allowed to take oral sips, when tolerated, within the first 24 hours, irrespective of the return of bowel functions. Carbohydrate-rich drinks were given to the patients to increase their caloric intake and hasten recovery. Early oral feeding was found to promote the return of GIT functions and to reduce the incidence of ileus and the risk of infections by improving the immunity [18, 19].

Although uncommon in surgical practice, fast track protocols in the literature did not recommend the routine use of abdominal drains following colorectal surgeries except in selective circumstances, such as severe bleeding and difficult dissection, and when they were used, they were removed as early as POD 1 [20] or POD 2 [11]. The mean time of removal of drains within the enhanced recovery group in our study was $2.94 \pm 1.06$ days compared to $9.06 \pm 3.76$ days in the conventional group. Although meta-analyses have reported no value of the routine use of drains for colorectal surgeries regarding the postoperative morbidities [21-23], we found that they were useful in detecting anastomotic leaks. Six out of 8 patients, in both groups, with intestinal leakage showed intestinal contents in the drains.

The overall postoperative complications in our study were relatively high compared with those of the studies reported in the literature because minor complications, such as mild wound infection and vomiting, were taken into account. Nonetheless, owing to the utilization of an evidence-based set of elements in the protocols of the enhanced recovery group, we observed a remarkable reduction in the incidence of these complications $(25.7 \%$ in the enhanced recovery group vs. $65.7 \%$ in the conventional recovery group, $\mathrm{P}=0.001)$. Certain morbidities, such as wound infection ( $\mathrm{P}$ $<0.001)$, respiratory tract infections $(\mathrm{P}=0.025)$ and anastomotic leakage $(\mathrm{P}=0.05)$, were significantly reduced in the enhanced group. Similarly, Greco's meta-analysis of 16 randomized controlled trials reported a significant reduction in the overall morbidity $(\mathrm{P}=0.001)$. However, it only showed a remarkable decrease in the nonsurgical complications $(\mathrm{P}<0.001)$ while the reduction in surgical complications was insignificant $(\mathrm{P}=0.13)$ [12].

Readmission of a patient was defined as hospitalizing that pa- 
tient for at least one day after successful discharge. In this study, the reason for readmission was either anastomotic leakage, abdominal wall dehiscence, intra-abdominal collection, or persistent PONV. The difference between the groups in our study was not significant $(\mathrm{P}=1.000)$, which was also the result when compared to different clinical trials applying these fast track protocols $[10,11,24]$.

In conclusion, implementation of enhanced recovery protocols within the health care pathway in our oncology center was successful, leading to more rapid recovery and fewer incidences of complications amongst those patients undergoing major abdominal/pelvic surgeries. The implementation of these protocols allowed earlier restoration of gut functions and faster hospital discharge while having no effect on the re-admission or the intrahospital mortality rate.

\section{CONFLICT OF INTEREST}

No conflicts of interest relevant to this article were reported.

\section{REFERENCES}

1. Wind J, Polle SW, Fung Kon Jin PH, Dejong CH, von Meyenfeldt MF, Ubbink DT, et al. Systematic review of enhanced recovery programmes in colonic surgery. Br J Surg 2006;93:800-9.

2. Debarros M, Steele SR. Perioperative protocols in colorectal surgery. Clin Colon Rectal Surg 2013;26:139-45.

3. Al Chalabi H, Kavanagh DO, Hassan L, Donnell KO, Nugent E, Andrews E, et al. The benefit of an enhanced recovery programme following elective laparoscopic sigmoid colectomy. Int J Colorectal Dis 2010;25:761-6.

4. Vlug MS, Bartels SA, Wind J, Ubbink DT, Hollmann MW, Bemelman WA, et al. Which fast track elements predict early recovery after colon cancer surgery? Colorectal Dis 2012;14:1001-8.

5. Francis N, Kennedy RH, Ljungqvist O, Mythen MG, editors. Manual of fast track recovery for colorectal surgery. Berlin: Springer-Verlag; 2012.

6. Varadhan KK, Neal KR, Dejong CH, Fearon KC, Ljungqvist O, Lobo DN. The enhanced recovery after surgery (ERAS) pathway for patients undergoing major elective open colorectal surgery : a meta-analysis of randomized controlled trials. Clin Nutr 2010;29: 434-40.

7. Kehlet H, Wilmore DW. Evidence-based surgical care and the evolution of fast-track surgery. Ann Surg 2008;248:189-98.

8. Wang G, Jiang ZW, Xu J, Gong JF, Bao Y, Xie LF, et al. Fast-track rehabilitation program vs conventional care after colorectal resection: a randomized clinical trial. World J Gastroenterol 2011;17: 671-6.

9. Serclová Z, Dytrych P, Marvan J, Nová K, Hankeová Z, Ryska O, et al. Fast-track in open intestinal surgery: prospective randomized study (Clinical Trials Gov Identifier no. NCT00123456). Clin Nutr 2009;28:618-24.
10. Vlug MS, Wind J, Hollmann MW, Ubbink DT, Cense HA, Engel AF, et al. Laparoscopy in combination with fast track multimodal management is the best perioperative strategy in patients undergoing colonic surgery: a randomized clinical trial (LAFA-study). Ann Surg 2011;254:868-75.

11. García-Botello S, Cánovas de Lucas R, Tornero C, Escamilla B, Espí-Macías A, Esclapez-Valero P, et al. Implementation of a perioperative multimodal rehabilitation protocol in elective colorectal surgery. A prospective randomised controlled study. Cir Esp 2011;89:159-66.

12. Greco M, Capretti G, Beretta L, Gemma M, Pecorelli N, Braga M. Enhanced recovery program in colorectal surgery: a meta-analysis of randomized controlled trials. World J Surg 2014;38:153141.

13. Alfonsi P, Slim K, Chauvin M, Mariani P, Faucheron JL, Fletcher D, et al. French guidelines for enhanced recovery after elective colorectal surgery. J Visc Surg 2014;151:65-79.

14. Compagna R, Aprea G, De Rosa D, Gentile M, Cestaro G, Vigliotti $G$, et al. Fast track for elderly patients: is it feasible for colorectal surgery? Int J Surg 2014;12 Suppl 2:S20-2.

15. Gustafsson UO, Scott MJ, Schwenk W, Demartines N, Roulin D, Francis N, et al. Guidelines for perioperative care in elective colonic surgery: Enhanced Recovery After Surgery (ERAS $\left(^{\circledR}\right)$ ) Society recommendations. World J Surg 2013;37:259-84.

16. Hughes M, Coolsen MM, Aahlin EK, Harrison EM, McNally SJ, Dejong $\mathrm{CH}$, et al. Attitudes of patients and care providers to enhanced recovery after surgery programs after major abdominal surgery. J Surg Res 2015;193:102-10.

17. Verma R, Nelson RL Prophylactic nasogastric decompression after abdominal surgery. Cochrane Database Syst Rev 2007;(1): CD004929.

18. Lee TG, Kang SB, Kim DW, Hong S, Heo SC, Park KJ. Comparison of early mobilization and diet rehabilitation program with conventional care after laparoscopic colon surgery: a prospective randomized controlled trial. Dis Colon Rectum 2011;54:21-8.

19. Ren L, Zhu D, Wei Y, Pan X, Liang L, Xu J, et al. Enhanced recovery after surgery (ERAS) program attenuates stress and accelerates recovery in patients after radical resection for colorectal cancer: a prospective randomized controlled trial. World J Surg 2012; 36:407-14.

20. Yang D, He W, Zhang S, Chen H, Zhang C, He Y. Fast-track surgery improves postoperative clinical recovery and immunity after elective surgery for colorectal carcinoma: randomized controlled clinical trial. World J Surg 2012;36:1874-80.

21. Rolph R, Duffy JM, Alagaratnam S, Ng P, Novell R. Intra-abdominal drains for the prophylaxis of anastomotic leak in elective colorectal surgery. Cochrane Database Syst Rev 2004;(1):CD002100.

22. Karliczek A, Jesus EC, Matos D, Castro AA, Atallah AN, Wiggers T. Drainage or nondrainage in elective colorectal anastomosis: a systematic review and meta-analysis. Colorectal Dis 2006;8:25965.

23. Tsujinaka S, Kawamura YJ, Konishi F, Maeda T, Mizokami K. Pel- 


\section{Coloproctology Mosab Shetiwy, et al.}

vic drainage for anterior resection revisited: use of drains in anastomotic leaks. ANZ J Surg 2008;78:461-5.

24. Wang G, Jiang ZW, Zhao K, Gao Y, Liu FT, Pan HF, et al. Fast track rehabilitation programme enhances functional recovery after laparoscopic colonic resection. Hepatogastroenterology 2012; 59:2158-63. 